\title{
Real-Space Mapping of the Two-Dimensional Phase Diagrams in Attractive Colloidal Systems
}

\author{
Bo Li,${ }^{1,3, *}$ Xiuming Xiao, ${ }^{1}$ Shuxia Wang, ${ }^{1}$ Weijia Wen, ${ }^{2,1}$ and Ziren Wang $\oplus^{1, \dagger}$ \\ ${ }^{1}$ Key Laboratory of Soft Condensed Matter Physics and Smart Materials, College of Physics, \\ Chongqing University, Chongqing, 401331, China \\ ${ }^{2}$ Department of Physics, Hong Kong University of Science and Technology, \\ Clear Water Bay, Hong Kong, China \\ ${ }^{3}$ Center for Soft and Living Matter, Institute of Basic Science, Ulsan, 44919, South Korea
}

(Received 29 March 2019; revised manuscript received 24 June 2019; published 22 August 2019)

\begin{abstract}
Phase diagrams reflect the possible kinetic routes and guide various applications such as the designed assembly and synthesis of functional materials. Two-dimensional (2D) materials have been a focus of ongoing research due to their wide applications; therefore, researchers are highly motivated to discover the general principles that control the assembly of such materials. In this study, we map the 2D phase diagrams of short- and long-range attractive colloids at single-particle resolution by video microscopy. Phase boundaries, including (meta)critical or triple points and corresponding real-space configurations, are precisely specified. Profiles of 2D phase diagrams with attractive interactions resemble their 3D counterparts. For short-range systems, by measuring the deepest achievable supercooled states on the phase diagram, a "crater" structure surrounding the metastable fluid-fluid critical point indicates an enhanced nucleation rate within the crater and further suggests a local minimum of the free-energy barrier for crystallization in this area. During a dense fluid-mediated two-step crystallization process, we observe that multiple crystallites could form within a single dense fluid cluster, partly due to its highly amorphous shape. This highly amorphous shape is found for all observed well-developed dense fluid clusters. It is a supplement to the multistep nucleation process. For long-range systems, equilibrium vapor-liquid coexistence is observed, which paves the way for the exploration of critical behaviors. Rigidity percolation of crystallites, and bulk fluid-solid coexistence which provide clear evidence of a possible first-order transition for 2D melting, are observed for both systems. Our experiments reveal the general features of phase behaviors shared by 2D attractive systems including graphene, protein membranes, and adsorbed nanocrystals.
\end{abstract}

DOI: 10.1103/PhysRevX.9.031032

\section{INTRODUCTION}

Over the years, the study of two-dimensional (2D) phase diagrams has been a perennial research focus [1-5], because such diagrams demonstrate the generic features of phase behaviors in highly functional materials including graphene, protein membranes, and nanocrystals on graphite. Although numerous theoretical discussions and simulations have been published, comprehensive experimental investigations into the phase behaviors of attractive 2D systems have been rare, partly because ergodic thermodynamic variables are difficult to obtain for the whole phase diagram.

\footnotetext{
*libotc@gmail.com

†wziren@cqu.edu.cn
}

Published by the American Physical Society under the terms of the Creative Commons Attribution 4.0 International license. Further distribution of this work must maintain attribution to the author(s) and the published article's title, journal citation, and DOI.
Subject Areas: Materials Science, Soft Matter, Statistical Physics
For long-range attractive systems, Nelson and Halperin [6] construct a speculative 2D phase diagram where solid, fluid, vapor, and hexatic phases are presented. This diagram is notably different from a three-dimensional (3D) diagram; it predicts that melting could proceed through two secondorder phase transitions from the solid to a hexatic phase and then from the hexatic to a liquid phase. Regarding a repulsive system, the transition order is proven to depend on the softness of the pair potential $[7,8]$. Compared with repulsive systems, attractive systems are more analogous to real materials and have been more intensively studied with simulations [9-14] and tested with several theories [15-17], but the topic of transition order remains a subject of debate $[2,18]$. Moreover, although widely reported in simulations, equilibrium 2D vapor-liquid coexistence has not heretofore been observed in colloidal experiments due to the challenges of realizing the long-range attraction. Because of well-known difficulties with the interpretation of simulation results in the vicinity of a second-order transition, published studies have arrived at inconclusive estimates of the critical temperature of the Lennard-Jones 

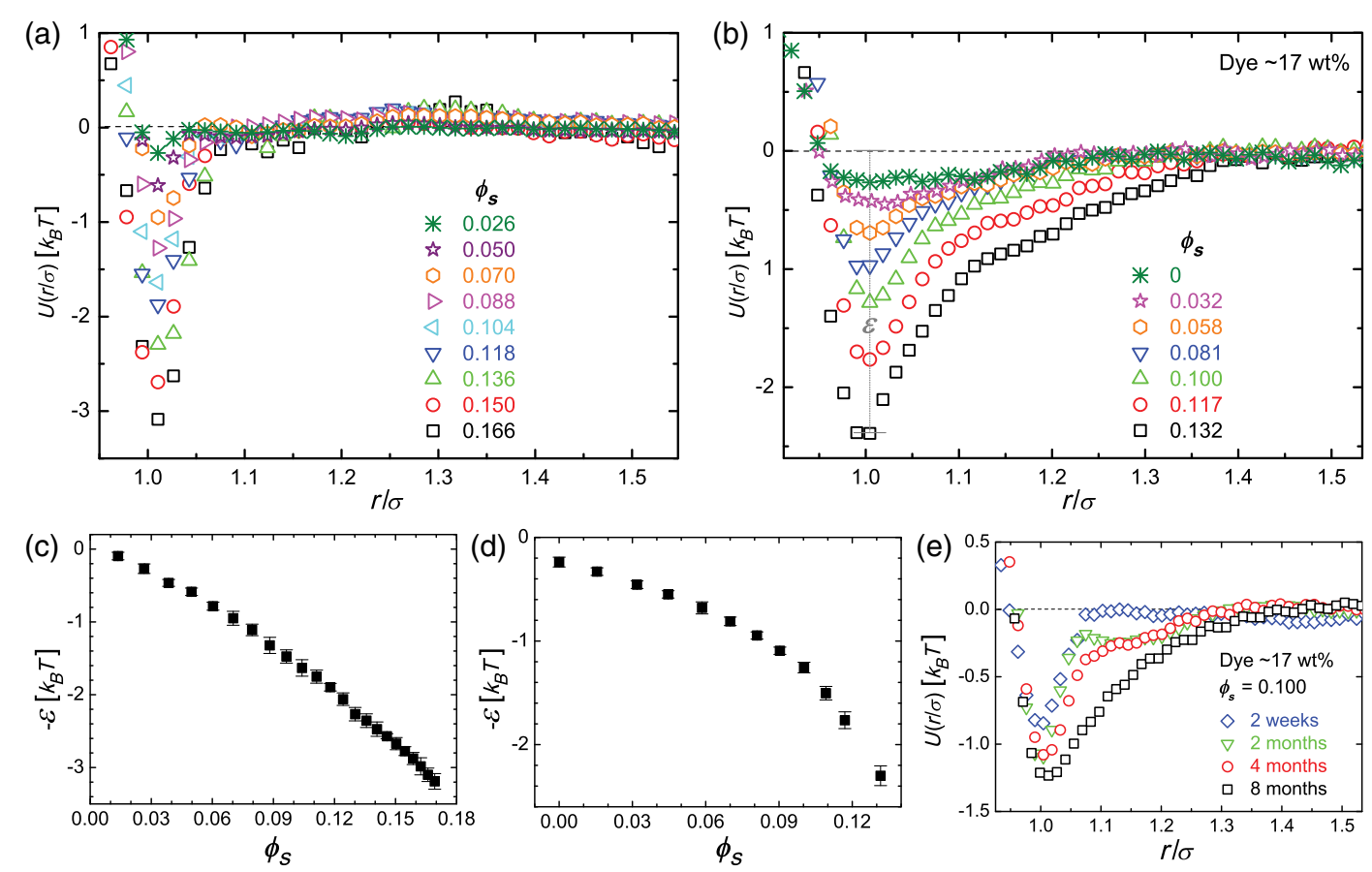

FIG. 1. Pair potentials of short-range (left) and long-range (right) attractions. Interparticle distance $r$ is normalized by the diameter of particle $\sigma . k_{B}$ is the Boltzmann constant, and $T$ is the Kelvin temperature. Attraction strength is defined as the depth of potential well $\varepsilon$, and $\phi_{s}$ denotes the volume fraction of small depletion particles. The attraction ranges are approximately $6 \%$ and approximately $33 \%$ for short- (a) and long-range (b) attraction systems, respectively. (c) and (d) denote the attraction strength as a function of the volume fraction of depletion particles for short- and long-range systems, respectively. (e) denotes the shape evolution of the long-range potential induced simultaneously by dye and small depletion particles. Please refer to the Appendix B for the measurement of the pair potential.

system $[19,20]$. Therefore, unequivocal observation of equilibrium vapor-liquid coexistence and extrapolation of the coexistence line are crucial for the decisive determination of the vapor-liquid critical point.

For systems with short-range attraction, such as membrane proteins confined in a quasi-2D geometry, Noro and Frenkel [13] reveal that the vapor-liquid transition becomes metastable with respect to the freezing transition when the attraction range is less than $15 \%$ of the diameter [5,21]. The universality of this attraction range effect on phase diagrams has been repeatedly strengthened in 3D systems [3-5,22-26]. Moreover, a simulation [3] proves that the free-energy barrier for homogeneous crystal nucleation reaches a minimum near the metastable critical point due to an enhanced density fluctuation, accompanied by a dense fluid-mediated two-step nucleation process. This multistep pathway verifies the Ostwald rule of stages [27] and is supported by simulations [28-30] and theoretical arguments [31,32]. Experimentally, the intermediate dense fluid is observed by dynamic light scattering in $3 \mathrm{D}$ [33-35] and by video microscopy in 2D systems [36,37].

The observation of an intermediate dense fluid does not, however, prove a local energy minimum near the (metastable) critical point, because the intermediate dense fluid could also be observed far away from the (metastable) critical point within the fluid-fluid regime in our experiment. Moreover, a dense fluid may offer an intrinsic free-energy advantage for crystallization of a hard sphere [29] where the (metastable) critical point does not exist and even appears below the triple temperature in a Lennard-Jones simulation [28]. Additionally, because nucleation experiments test only one possible route on the phase diagram plane, the exact condition under which two-step nucleation occurs remains elusive $[18,33]$. At last, to offer a clear insight into the role that the metastable fluid-fluid regime plays in the assistance of crystallization nucleation, it is desirable, under some conditions, to witness a full fluid-fluid phase separation with any given shape of droplets of dense fluid, instead of a dense region, inside the dilute fluid before crystallization begins $[18,30]$.

To address the aforementioned questions, we map the phase diagrams of colloids with short- and long-range attractions by video microscopy. Because the motion of each particle can be directly seen with an optical microscope, colloids as an outstanding model system are used in phase diagram drawing for 3D experimental systems composed of hard spheres $[38,39]$ and repulsive particles [40] under magnetic or electric fields. In this article, we systematically investigate how the attraction range influences the phase behaviors. Positions of phase boundaries including critical and triple points are precisely specified. Real-space observations of evolving structures and configurations of each phase are captured down to the single-particle level. Specifically, for short-range systems, 

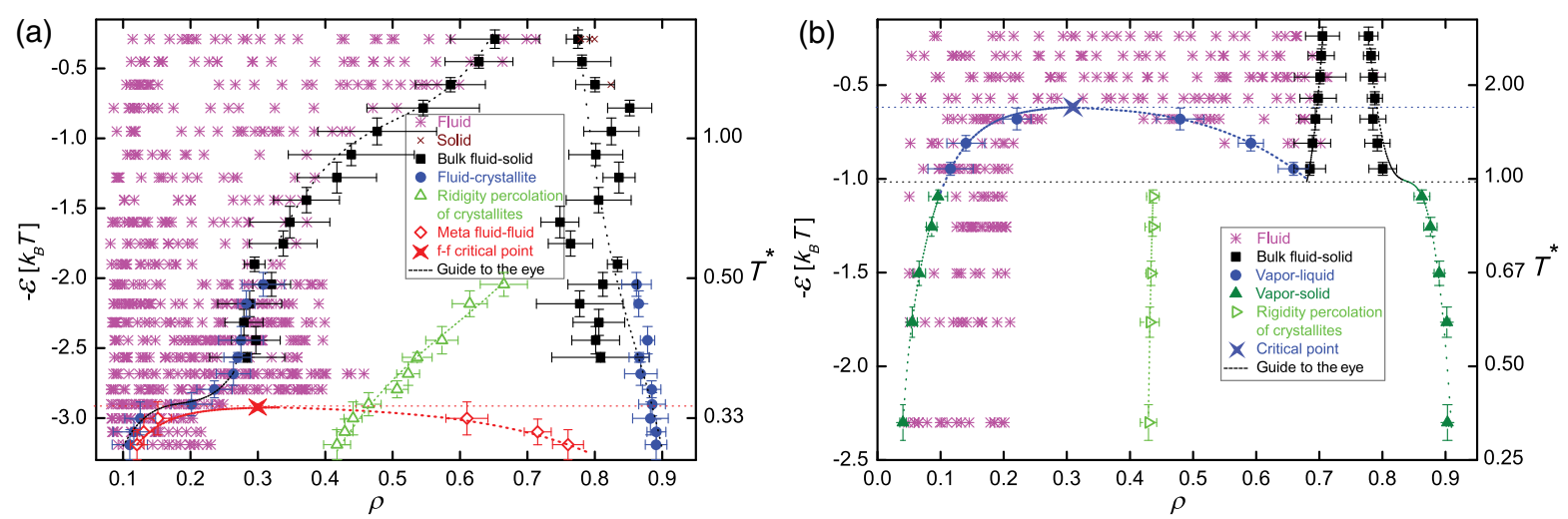

FIG. 2. Short-range and long-range phase diagrams in attraction strength $\varepsilon$-areal density $\left(\rho=\left[\left(\pi \sigma^{2} n\right) / 4\right]\right)$ representation. $n$ is the number density, and $\sigma$ is the diameter of particles. Refer to Fig. 3 for the corresponding real-space configuration of each coexistence.
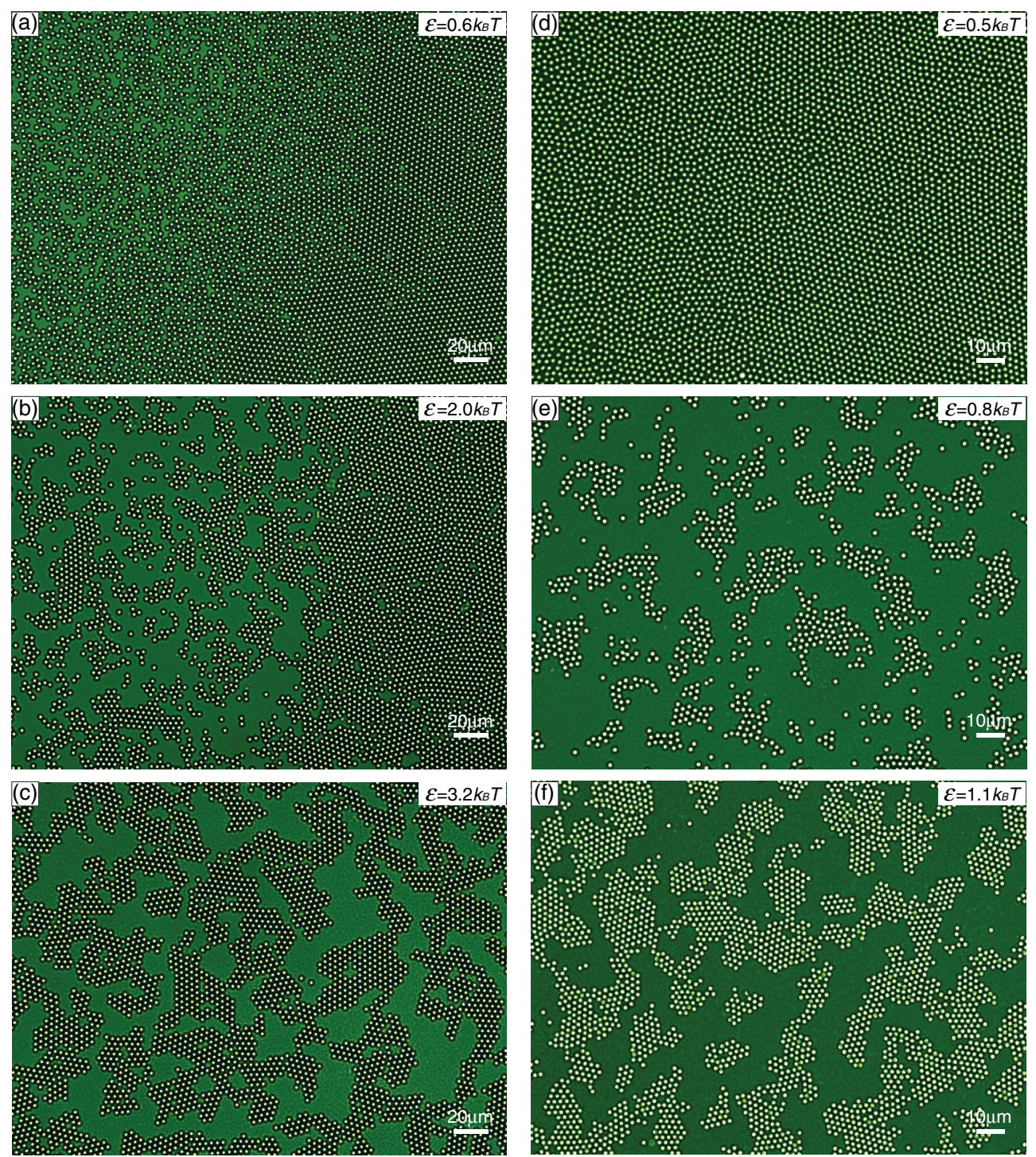

FIG. 3. Typical real-space images for coexistence states of (a)-(c) short- and (d)-(f) long-range systems. (a),(d) Bulk fluid-solid coexistence. (b) Bulk fluid-solid + fluid-crystallite coexistence. (c),(f) Crystallites in contact with each other become a rigidity percolation state. (e) Vapor-liquid coexistence. Please refer to Appendix D for the identification criteria of each phase and the corresponding movies. 
by measurement the achievable supercooled states, a "crater" structure surrounding the metastable critical point on the phase diagram indicates an enhanced nucleation rate for homogeneous crystallization within the crater. The dense fluid clusters inside a dilute fluid are in a highly amorphous shape, and multiple crystallites are found to evolve within a single fluid cluster during a dense fluidmediated two-step crystallization process. This evolution is a supplement to the multistep nucleation process. For long-range systems, stable vapor-liquid coexistence is observed, which is crucial to addressing questions regarding poorly understood critical behaviors.

\section{THE EXPERIMENTAL SYSTEM}

Inducing long-range attractive interaction between colloidal particles is challenging. Recently, a long-range attraction was proposed between colloidal polymethylmethacrylate (PMMA) particles by adding a type of nonfluorescent dye into an aqueous colloidal suspension. In contrast to the previous long-range attractive system [41], the strength of the dye-induced attraction can be precisely tuned. However, attraction strength induced purely by the dye is limited to $0.6 k_{B} T$, which is too small to allow the observation of vapor-liquid coexistence. Later, a tunable anharmonic attraction between paramagnetic particles that are confined within 2D was generated using a highfrequency rotating magnetic field [42]. By adjusting the strength of the interaction in a wide range, fluidlike clusters and crystallites with hexagonal ordering are observed. Here, we advance a new technique by simultaneously adding the nonfluorescent dye and smaller depletant PMMA (Microparticles $\mathrm{GmbH}$ ) particles of diameter $\sigma=$ $0.192 \mu \mathrm{m}$ into a colloidal suspension with large PMMA particles (Microparticles $\mathrm{GmbH}$ ) of $\sigma=2.27 \mu \mathrm{m}$. We find that the dye is able to modulate the short-range depletantinduced attraction into a long-range attraction. As shown in Fig. 1(e), the system undergoes a complex evolution process. The range of attraction increases with time and reaches a steady state 8 months after preparation. During the same period, the attraction strength is also enhanced by approximately $40 \%$ after 8 months comparing that measured after 2 weeks. Note that both the range and strength of the attraction remain stable for the 24-48 h during which we conduct the experiments. The maximum attraction strength (approximately $2.3 k_{B} T$ ) used in our experiment is located deep in the vapor-solid regime [Figs. 1(b) and 1(d) and solid triangles in Fig. 2(b)]. The short-range depletion attraction [43] is obtained by adding different quantities of $0.192 \mu \mathrm{m}$ PMMA (Microparticles $\mathrm{GmbH}$ ) particles into $3.27 \mu \mathrm{m}$ PMMA particles (Microparticles $\mathrm{GmbH}$ ) [Fig. 1(a)].

We construct the phase diagrams in terms of attraction strength $(\varepsilon)$-areal density $(\rho)$ representation. For each sample with a particular $(\rho, \varepsilon)$ pair, we start to collect data after $2-5 \mathrm{~h}$ equilibration. For each $(\rho, \varepsilon)$ pair, 6-10 samples are checked to ensure the consistency of the phenomenon. The phase boundaries are obtained by respectively measuring the density of each phase at coexistence (Fig. 3). For more details of this experimental system, please refer to Appendix A.

\section{RESULTS}

\section{A. Phase diagrams for short- and long-range 2D attractive systems}

As illustrated by Fig. 2, 2D phase diagrams are qualitatively similar to their 3D counterparts, which agrees with previous studies $[4,13,22,44-46]$. The phase behavior in $2 \mathrm{D}$ is sometimes markedly different from its $3 \mathrm{D}$ counterpart for nontrivial reasons (such as melting via an intermediate hexatic phase from crystals to liquids). In other cases, 2D and 3D phase behaviors are similar, but this similarity is difficult to prove experimentally. Our results directly demonstrate that dimensionality does not alter the shape of the phase diagram, implying that more fundamental statistical rules control the phase transitions. Both short- and long-range systems demonstrate obvious bulk fluid-solid (triangular lattices) coexistence at $0.3 k_{B} T \leq \varepsilon \leq 1.9 k_{B} T$ [solid squares in Figs. 2(a) and 3(a) and Supplemental Material [47], Movie 1] for short-range systems and $0.2 k_{B} T \leq$ $\varepsilon \leq 1.0 k_{B} T$ [solid squares in Figs. 2(b) and 3(d) and Supplemental Material [47], Movie 5] for long-range systems, respectively. This observation serves as clear experimental evidence of a first-order transition between a fluid and a solid, a finding that would be trivial in 3D but not in $2 \mathrm{D}$.

For a short-range system, when a fluid slightly crosses over the phase boundary, it becomes supercooled and quickly relaxes towards equilibrium through heterogeneous crystallization nucleation due to the wetting effect at the edge, forming a bulk crystalline phase through epitaxial growth. Bulk solids are always in the form of polycrystals rather than large domains of single crystals. Grain boundaries between polycrystalline domains disrupt the quasilong-range orientational order and preclude the identification of possible hexatic phases. We generally count a phase as a solid phase (be it hexatic or polycrystalline) when the solid area is larger than 300 layers (approximately 5 field of view). A solid exhibits bulk properties when its number of layers is larger than 50 (Fig. 7). Notably, the interface has a larger range of effect in a $2 \mathrm{D}$ system than that in a $3 \mathrm{D}$ system [48].

As strength rises, fluid density gradually decreases. For a fixed density of fluids, the long-time diffusion coefficient $D_{f}$ (Fig. 8) (i.e., dynamics of the system) decreases with the attraction strength $\varepsilon$. Mixture states of fluid-solid coexistence and fluid-crystallite coexistence [Fig. 3(b) and Supplemental Material [47], Movie 2] begin to appear at $\varepsilon \geq 2.0 k_{B} T$. The bulk crystallites have numerous voids [Fig. 3(b)], which is a typical structural feature of an attractive system. However, the overall density of the solid almost remains constant [Fig. 2(a)], since strong attraction tends to make a relatively compact crystallite, compromising the free volume induced by the voids. 

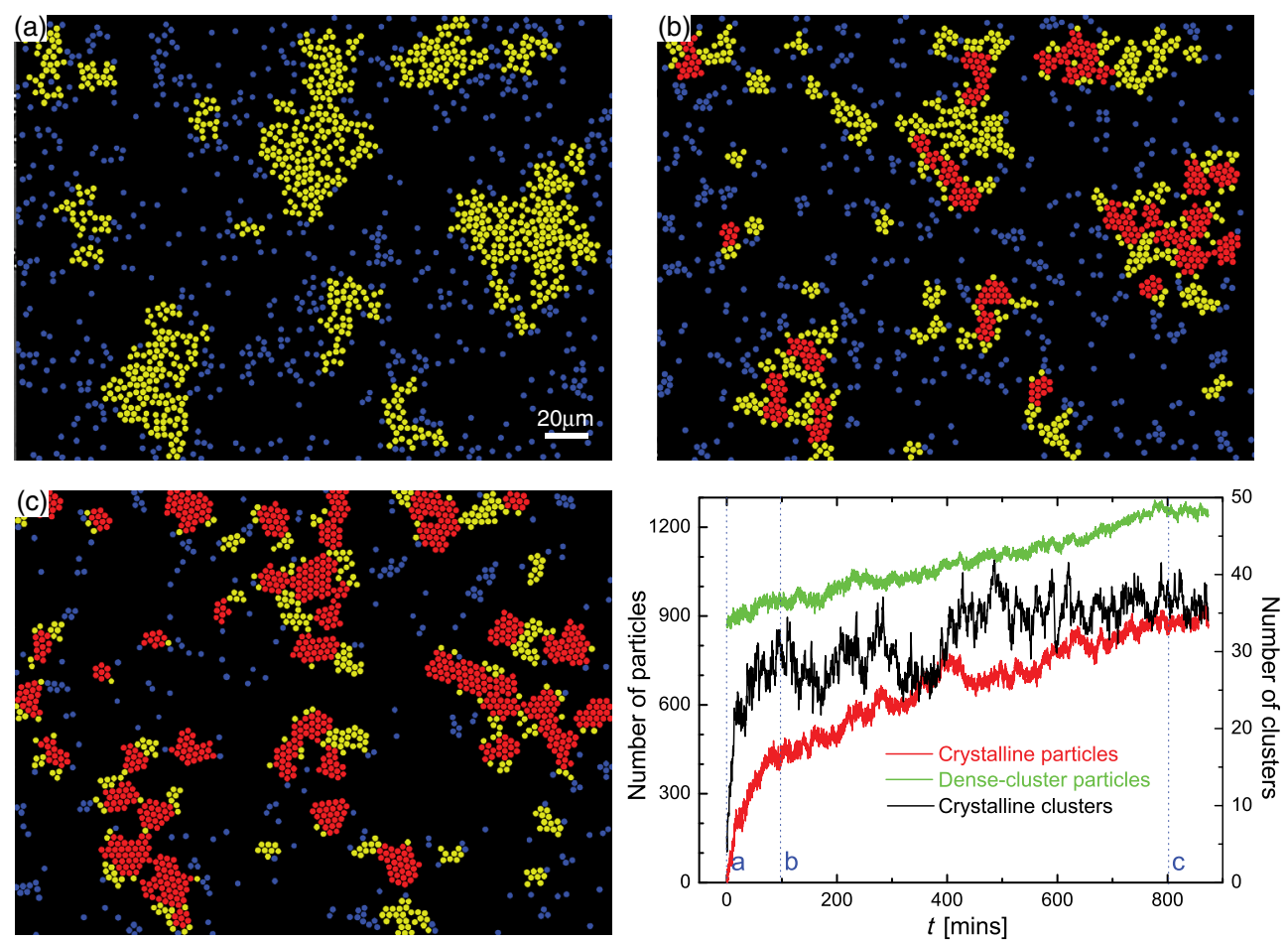

FIG. 4. A full metastable fluid-fluid separation in large scale (a) relaxing, (b) into equilibrium vapor-solid coexistence, (c) at $\varepsilon=3.0 k_{B} T$. Each particle is colored by the instant structure it is in. Blue, particles within lower-density fluid clusters defined by the local areal density (ratio of areas of particles and voronoi cells) less than 0.5; yellow, particles within high-density fluid clusters (including interfacial particles at the edge); red, particles in crystalline structures (including interfacial particles at the edge) defined by conventional local orientational order parameters $\left|\psi_{6 j}\right| \equiv\left|\left(1 / Z_{j}\right) \sum_{k=1}^{z_{j}} e^{6 i \theta_{j k}}\right| \geq 0.8$, where $\theta_{j k}$ denotes the angle of the bond between the reference particle $j$ and its neighbor $k . Z_{j}$ is the number of nearest neighbors identified from Delaunay triangulation. Particles are considered to be connected and to belong to the same cluster when the distance is less than 1.5 $\sigma$ [3]. (d) Evolution of the total number of crystalline and dense-cluster particles with time.

As the dynamics further weaken, when $\varepsilon \geq 2.7 k_{B} T$, the system is totally in a fluid-crystallite coexistence (i.e., in a configuration of dispersed crystallites with fluid) (Fig. 10). Averaged translational diffusion coefficients $D_{f}^{T}$ for all crystallites (as a whole rigid object) decay with the size (Fig. 9 and Appendix C) until they are in connection with each other and percolate the system, reaching an immobile rigidity percolation state [49] [open triangles in Figs. 2(a) and 3(c) and Supplemental Material [47], Movie 3]. Although the crystallites are in contact with each other, each crystallite exhibits its distinct ordering orientation. The rigidity percolation differs from the percolation in simple fluids [50] because of its frozen dynamics (Fig. 9). It is also distinct from the percolation frequently observed in 3D attractive colloidal systems [4,51,52]. In these systems, when the attraction is strong enough, gelation occurs and leads to a stringlike disordered morphology. In our experiment, the percolated state features well-ordered crystallites. By contrast, the ordered percolated state in our work is more similar to the network of microcrystalline clusters observed in 3D PMMA colloids with competing long-range repulsions and short-range attractions when the attraction strength is moderately strong [53]. Because the crystallites possess a finite size and random orientation, the peaks in the radial distribution function (Fig. 6) exhibit a dramatic decay with increasing distance and almost vanish when the distance exceeds $5 \sigma$, which is qualitatively consistent with that observed in Ref. [53]. This result suggests that the system can be viewed as a homogeneous phase when the scale length exceeds $5 \sigma$. In brief, due to the fact that the percolated crystallites cannot fill the whole space and the lack of long-range orientational order, the rigidity percolation state observed in our experiment is a special crystal phase. Its features and formation mechanism are worth more exploration.

When $\varepsilon \approx 2.9 k_{B} T$, metastable fluid-fluid coexistence [open diamonds in Figs. 2(a) and 4 and Supplemental Material [47], Movie 4] occurs immediately after a rapid decrease in the liquid areal density, at which the freezing line [dashed line in Fig. 2(a)] is shaped as the "shoulder" which has been introduced and discussed in a previous 2D simulation [13]. According to the simulation [13], the shoulder is found to appear at $\varepsilon$ of $2.6 k_{B} T$ to $3.1 k_{B} T$, depending on the range of the short-range attraction force $(\leq 15 \%)$. Our measured position $2.9 k_{B} T$ falls in this range of $2.6 k_{B} T$ to $3.1 k_{B} T$. 
Unlike a short-range system, the difference in areal densities between the fluid and solid for a long-range system only slightly increases with $\varepsilon$ in the whole regime of bulk fluid-solid coexistence. Most importantly, we discover vaporliquid coexistence when $\varepsilon$ is between $0.7 k_{B} T$ and $1.0 k_{B} T$ [solid circles in Figs. 2(b), 3(e), and 11 and Supplemental Material [47], Movie 6]. Vapor-liquid separation can last for months, meaning that it is indeed an equilibrium state. The vapor-liquid interface is rough and foamy, which implies a low value of line tension. Theoretically, a small line tension leads to a low-energy barrier for the condensation transition. This low-energy barrier, as well as the previous simulation results $[54,55]$, is in consistency with the fact that we fail to observe the coexistence of bulk vapor and liquid phases due to the nucleation events that frequently occur throughout the system.

When $\varepsilon \geq 1.0 k_{B} T$, a typical system crosses over the triple points and enters the vapor-solid coexistence regime [solid triangles in Fig. 2(b)]. We always observe dispersed crystallites instead of bulk solids in this regime [Fig. 3(f)]. Similar to the situation in a short-range system, crystallites ultimately make contact with each other, forming an ordered percolated state on the scale of the whole system [Fig. 3(f) and Supplemental Material [47], Movie 7]. In comparison with the rough interface of vapor-liquid coexistence, it is qualitatively indicated that line tension $\tau_{\text {vapor-solid }}>\tau_{\text {vapor-liquid }}$ [Figs. 3(e) and 3(f) and Supplemental Material [47], Movies 6 and 7].

The precise location of the critical point and triple points is crucial to study the universality of a long-range LennardJones system. However, the exact position of phase boundaries is still a subject of some debate [55]. The reduced temperatures $\left(T^{*}=k_{B} T / \varepsilon\right)$ for the critical point $T_{c}^{*}$ and triple points $T_{t}^{*}$ are $0.4-0.7$ and approximately 0.4 , respectively, given by different simulations of the LennardJones system [10,19,56-58]. However, the extrapolated critical and triple temperatures in our experiments [broken line in Fig. 2(b)] are at approximately $1.5\left(\varepsilon=0.7 k_{B} T\right)$ and approximately $1.0\left(\varepsilon=1.0 k_{B} T\right)$, respectively, approximately twice the values in Lennard-Jones simulations. Although our long-range potential is different from the Lennard-Jones potential, the long-distance attractive part of our long-range potential can be adequately fitted by a power-law function (Fig. 12 and Appendix E), revealing its long-range nature. Because we still observe all phase behaviors from previous computational short- and longrange phase diagrams $[3-5,13,21,22]$, the specific form of the potential chosen to study phase behavior is seemingly rendered qualitatively unimportant. However, quantitative results such as critical or triple temperatures may strongly depend on the detailed form of potentials. In addition to the range of attractive interactions, many other aspects, such as the softness of the repulsive part or the cutoff value, can alter the local arrangement of the particles and, thus, influence the phase in equilibrium. Our observation thus implies that more exploration is required of the key effects that cause this quantitative discrepancy between our experiment and previous simulations.

Although inconsistent in terms of attraction strength, all areal densities for the critical point $(\rho \approx 0.35)$ and triple points ( $\rho s \approx 0.10,0.67,0.90$ ) [Fig. 2(b)] in our experiment are close to the values reported from simulations $[9,19,58]$ and from a nucleation experiment [36]. This observation, therefore, suggests that characteristic areal densities are not sensitive to the details of the pair potential.

Although the distinct profiles of the phase boundaries, short-range and long-range systems share some common features, which are probably general for all attractive systems. First, both the (metastable) critical points are at the same density $(\rho \approx 0.35)$, which suggests that areal densities of (metastable) critical points may not be sensitive to the range of attractive interactions. Second, as illustrated in Figs. 2(a) and 2(b) (symbol in open triangles), areal densities for rigidity percolation in a large- $\varepsilon$ regime saturate at the same value of $\rho=0.42$ for both systems. In addition, we observe highly anisotropic crystallites with sharpfaceted interfaces along the $\langle 10\rangle$ direction. Therefore, the (11) lattice has the minimal line tension $\tau$ for 2D triangular lattice crystallites. Finally, the width of transition layers between the bulk solid and bulk fluid or vapor decreases with $\varepsilon$ for both systems.

\section{B. Enhanced nucleation rate around $T_{c}$ and metastable dense fluid-mediated two-step nucleation}

For short-range attraction, the equilibrium vapor-liquid transition is preempted by freezing, becoming a metastable fluid-fluid state. In particular, Wolde and Frenkel [3] show that the presence of the metastable critical point will lower the homogeneous barrier for crystal nucleation, due to the strong density fluctuation. Here, we confirm this argument by measuring the deepest achievable supercooled fluid state within the field of view and on one day [double cross in Fig. 2(a)], which is equivalent to the induction time (i.e., inversely proportional to the nucleation rate) for homogeneous nucleation. As indicated by Fig. 2(a), the configuration of the deepest achievable supercool liquid manifests a crater structure near the extrapolated metastable critical point $\left(\varepsilon=2.93 k_{B} T\right)$. The nucleation rate should be approximately the same on the edge of the craterlike structure. This structure indicates that the supercooling capacity declines at approximately the critical point, causing the nucleation rate to increase within the crater. Because the nucleation rate is determined through an exponential relationship by the height of the energy barriers for a corresponding system, the crater structure on the phase diagram indicates a free-energy minimum near $T_{c}$.

Reduction of the achievable supercooled fluid state begins at $\varepsilon=2.9 k_{B} T$, slightly higher than the metastable fluid-fluid critical point $\varepsilon=2.93 k_{B} T$. This result is direct proof that the crystallization nucleation rate is enhanced 
by the critical fluctuations [3]. Therefore, searching for the fluid-fluid coexistence region to promote crystallization nucleation is unnecessary. Instead, the system can approach the metastable critical point even if it is outside the fluidfluid coexistence boundary. In this manner, the possible dense fluid caused by critical density fluctuation is unstable; thus, the two-step nucleation mechanism may operate in systems where no intermediate is independently found [25].

Figure 4 (Supplemental Material [47], Movie 4) demonstrates a 12-h relaxation process of dense-fluid clusters relaxing into crystallites when the system slightly enters a fluid-fluid regime $\left(\varepsilon=3.0 k_{B} T, \rho=0.17\right)$. Three large (size approximately 170), well-established dense-fluid clusters appeared before crystallization [Figs. 4(a) and 4(d)], which is in consistency with numerical predictions of a liquid aggregation consisting of a few hundred particles before crystallization [30]. Notably, the morphology of the dense-fluid cluster exhibits highly rough (possibly fractal) boundaries rather than spheres or polygons as commonly presumed. The transition between dense fluids and crystallites is a typical nucleation process that many transient nuclei with crystalline structures form and break up until the first nucleus reaches a critical size (Supplemental Material [47], Movie 4). Startlingly, we find that multiple crystallites can evolve from a single dense liquid cluster [Fig. 4(b)], partly because of the fractal-like morphology of the dense fluid cluster. Because of this multinucleation mechanism, we finally obtain several contacting crystallites [Figs. 4(a) and 4(c)] of different orientations. After $100 \mathrm{~min}$, the number of crystalline clusters saturates [Figs. 4(b) and 4(d)], after which the increases in crystalline particles are proportional to those of dense-cluster particles (dense-fluid particles + crystalline particles) $[3,37]$. Multiple nucleation within a single dense fluid gives rise to complex correlations between the size or morphology of the initial dense fluid and those of equilibrium crystallites. Our findings suggest that the kinetic pathway of dense-fluid-mediated nucleation is more complicated than had been expected and may offer a supplement to the two-step nucleation theory.

\section{DISCUSSION}

In summary, we mapped the $2 \mathrm{D}$ phase diagrams for colloidal systems with short-range and long-range attractive interactions by video microscopy. The configuration of each phase and relevant evolution were illustrated at singleparticle resolution for both diagrams. The range of interaction had a dramatic influence on the shape of the phase boundaries and, thus, the nature of related phase transitions. Meanwhile, both phase diagrams shared some features. For short-range systems, the crater structure of measured achievable supercooled states on a phase diagram indicates an enhanced nucleation rate within the crater and further suggests a local minimum of the free-energy barrier for crystallization in this area. Multiple crystallites were found to form within a single well-established dense fluid cluster with a highly amorphous shape, which provided experimental input in the further refinement of the two-step nucleation theory. For long-range systems, equilibrium vapor-liquid coexistence was observed, which paves the way for the exploration of critical behaviors. The observation of bulk fluid-solid coexistence for both systems shows the possibility of the first-order transition in melting of 2D systems. Rigidity percolation of crystallites was also observed for both systems at a deep attraction strength. Usually, biological and atomic or molecular systems, respectively, have short-range and long-range attractions, analogous to the interactions adopted in our experiments. Our experimental studies can provide insights into the kinetics of phase transformations and can improve the understanding of related biological and atomic or molecular processes.

The experimental system enabled us to explore the whole areas of 2D phase diagrams and to address crucial open questions such as those regarding critical behaviors that are difficult to measure in real atomic or molecular systems. For example, both our measured critical and triple temperatures were overestimated in comparison with the results reported by Lennard-Jones-based simulations; our result justifies more detailed investigations about the influence of the detailed form of the potential on the phase behaviors. Besides, combining imaging techniques with this platform will hopefully enable the direct observation of individual particles undergoing phase dynamics such as vapor-liquid transitions. It is worthwhile to apply the capillary wave model to quantify the fluctuation of $2 \mathrm{D}$ vapor-liquid interfaces like the interfacial tension, as it has been used to analyze the fluid-fluid interface in a phase-separated colloid-polymer dispersion with confocal microscopy [59]. Furthermore, it is also interesting to take a more quantitative analysis such as drawing the free-energy landscape on the process of multiple nucleation of crystallites inside a dense droplet. This analysis may reveal the secrets of complex pathways and may shed light on other debates.

Beyond 2D systems, our experiments also provided a platform for the study of phase behaviors of thin-film materials. Moreover, by adding nonspherical depletant particles, one can induce anisotropic attractive interactions at different ranges. How the phase diagram will be changed in systems with anisotropic interactions compared with that in isotropic systems is another interesting issue.

Besides crystallizing systems, it is worthwhile to test the phase behaviors of glass-forming systems based on our attractive colloidal systems. Topics like under what conditions the attractive interaction can induce phase separation in binary colloidal glasses and how the range of attractive interactions alters the fragility of the colloidal glasses are also interesting open questions.

Finally, the mechanisms through which long-range attraction is induced by the dye and why this attraction evolves with time remain unclear, meriting additional exploration into this system. 


\section{ACKNOWLEDGMENTS}

We thank Daan Frenkel for the insightful discussions. This study was supported by the National Natural Science Foundation of China (Grant No. 11604031) and the Fundamental Research Funds for the Central Universities of China (Grant No. 106112015CDJZR308801). B. L. acknowledges the support by the Korean Institute for Basic Science (Project Code IBS-R020-D1).

\section{APPENDIX A: EXPERIMENTAL METHODS}

\section{Preparation of long- and short-range attractions}

To induce long-range attraction, we prepare nonfluorescent liquid dye (D980101 Chromatint jet black 1990 Chromatech Incorporated) by volume fraction $60 \%$ (contains $17 \%$ solid dye by weight after drying). Then, precisely calculated amounts of PMMA particles (diameter $\sigma=0.192 \mu \mathrm{m}$ ) (Microparticles $\mathrm{GmbH}$ ) and PMMA $(\sigma=2.27 \mu \mathrm{m})($ Microparticles $\mathrm{GmbH})$ powders are added to the dye solution to tune the depth of the potential well and areal density, respectively. Other types of depletant particles such as $\mathrm{SiO}_{2}$ also apply to induce long-range attraction. Compared with the method of adding a particle solution, directly adding a powder into the dye solution prevents the change of dye concentration in the systems. Finally, we store the suspension at room temperature for approximately 8 months. Notably, small depletant particles do not suppress the crystallization of large particles, because depletant particles can remain in the central space constituted by close packing of three larger spheres.

For short-range attraction, the method that is applied resembles that applied for long-range attraction. Here, we add depletant PMMA particles $(\sigma=0.192 \mu \mathrm{m})$ (Microparticles $\mathrm{GmbH})$ into PMMA particles $(\sigma=3.27 \mu \mathrm{m})$ (Microparticles $\mathrm{GmbH}$ ). Because the PMMA particles we use are carboxyl functionalized, $3 \mathrm{mM}$ sodium dodecyl sulfate is added to screen the Coulomb electrostatic repulsion and make the potential approximately equal to that for hard + attractive interactions with a Debye length of approximately $20 \mathrm{~nm}$. The physical diameters of the large PMMA particles are calibrated so that the density in the closed packing monolayer is 0.907 .

\section{Experimental setup}

Large PMMA particles are strictly confined between two glass walls with little out-of-plane motion; particles always remain in the focal plane. Two glass surfaces are cleaned so that PMMA particles do not adhere to the surfaces. Because the diameters of small depletant particles are much smaller than the separation of the glass walls (i.e., the diameters of large PMMA particles), the volume fraction $\phi_{s}$ can be used to quantify the amount of depletant particles. Thus, the depletion attraction used here is, in fact, acting in 3D geometry, whereas the particles are confined to quasi-2D geometry, that resembles 3D colloid + polymer mixture systems $[22,60]$ but confined to 2D geometry. Data are acquired after an equilibration time of $2-5 \mathrm{~h}$ according to the diffusion coefficients (Fig. 8) measured at different $\varepsilon$. Spheres are uniformly distributed within a sample area of $\mathrm{mm}^{2}$, except for the phase separation and the areas near the edges. No obvious drift or flow is observed, which agrees with the observation of apparent stationary transition layers between the bulk fluid and bulk solid phases [Fig. 3(a)]. The areal density (i.e., the total number of particles) values in the field of view are almost the same during the measurements. Images are taken at (1-5) frames per second using an upright optical microscope at central area $70 \times 70 \sigma^{2}$. All the experiments are conducted at a constant room temperature of $24^{\circ} \mathrm{C}$. Sphere positions are located by widely adopted routines [61] written in Interactive Data Language. In total, we prepare thousands of samples and make approximately 2500 qualitative measurements in drawing both the phase diagrams (Fig. 2).

\section{APPENDIX B: MEASUREMENT OF RADIAL DISTRIBUTION FUNCTION AND PAIR POTENTIAL}

The radial distribution function $g(r)$ [Figs. 5(a)-5(c) and 6] is the probability of finding a pair of atoms a distance $r$ apart relative to the probability for a completely uniform distribution. For a 2D isotropic system, it can be easily measured, in principle, by counting pairs separated by $r$ to $r+d r$ and then normalizing by $2 \pi r n d r$, where $n=N / A$ is the areal density in a field of view containing $N=\langle N\rangle_{t}$ particles and $A$ is the area of the field of view.

Pair potential $U(r)$ is extracted by analyzing the radial distribution function $g(r)$ [Figs. 5(a)-5(c)] measured in a dilute fluids of a monolayer of spheres with the OrnsteinZernike integral equation of liquid-structural theory $[62,63]$. Here, the $g(r)$ are measured at density $\rho \approx 5 \%$ and $\rho \approx 10 \%$ for long- [Fig. 5(b)] and short-range [Fig. 5(a)] systems, respectively. Image artifacts [64] are corrected using the method described in Ref. [65]. Both the Percus-Yevick and hypernetted-chain approximation algorithms of liquid-structure theory produce error bars smaller than $0.1 k_{B} T$ [Figs. 1(c) and 1(d)] of $U(r)$ from sample to sample. 

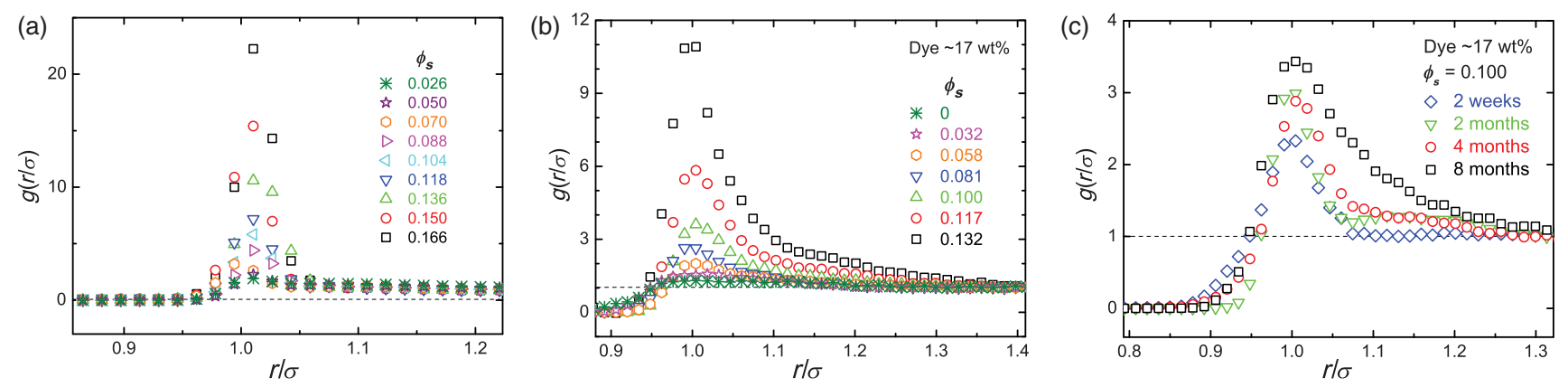

FIG. 5. (a) Radial distribution function of a dilute (areal density approximately 10\%) monolayer of $\sigma=3.27 \mu \mathrm{m}$ PMMA particles at various depletant concentrations for the short-range attractive pair potential measurement presented in Fig. 1(a). (b) Radial distribution function of a dilute (areal density approximately 5\%) monolayer of $\sigma=2.27 \mu \mathrm{m}$ PMMA particles at various depletant concentrations for the long-range attractive pair potential measurement illustrated in Fig. 1(b). The mass concentration of the dye is approximately $17 \%$. (c) Temporal evolution of the radial distribution function of a dilute (areal density approximately $5 \%$ ) monolayer of $\sigma=2.27 \mu$ m PMMA particles at depletant concentrations $\phi_{s}=0.100$ for the long-range attractive pair potential measurement presented in Fig. 1 (e). Interparticle distance $r$ is normalized by the diameter of particle $\sigma$.

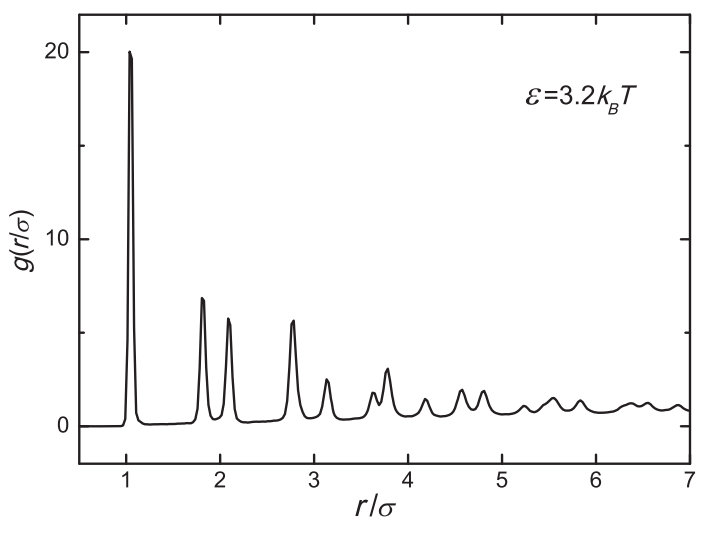

FIG. 6. Radial distribution function for a short-range system at the rigidity percolation point. The profile of the peaks of the radial distribution function decays rapidly and becomes even when the distance is larger than $5 \sigma$.

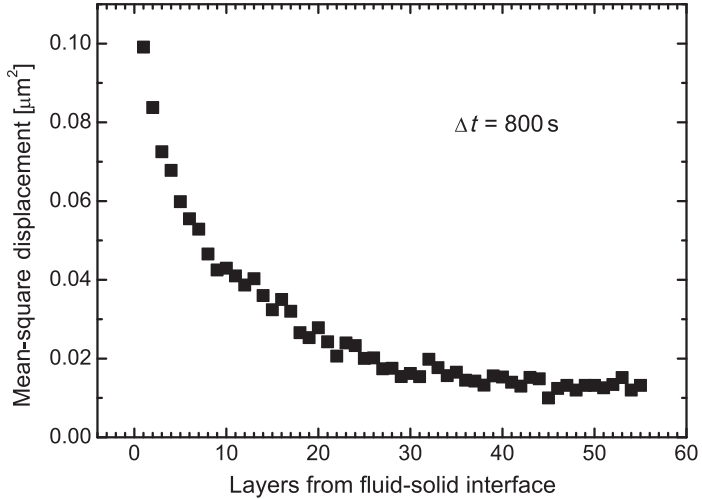

FIG. 7. Mean-square displacement of solids as a function of the layers from the bulk fluid-solid interface at time $800 \mathrm{~s}$ for a shortrange system at $\varepsilon=1.6 k_{B} T$. The fluid-solid interface is relatively sharp. The solid has bulk properties when the number of layers is larger than 50 from the interface. Please refer to Appendix $\mathrm{C}$ for calculation details.

\section{APPENDIX C: MEASUREMENT OF MEAN-SQUARE DISPLACEMENT AND LONG-TIME DIFFUSION COEFFICIENT}

The long-time diffusion coefficients (Figs. 8 and 9) are defined via $D=\{[\Delta(t)] / 4 t\}$, where $\Delta(t)=\left\langle\left[r_{i}(t)-r_{i}(0)\right]^{2}\right\rangle$ is the mean-square displacement. \langle\rangle denotes a canonical average for liquids or average of all crystallites in the field of view with the same size. $r_{i}$ denotes either the position of a given liquid particle $i$ or the position of the center of mass for a given crystallite $i$. The data at a long time part, where mean-square displacement exhibits a linear relationship with $t$, are used in the calculation of diffusion coefficients. 

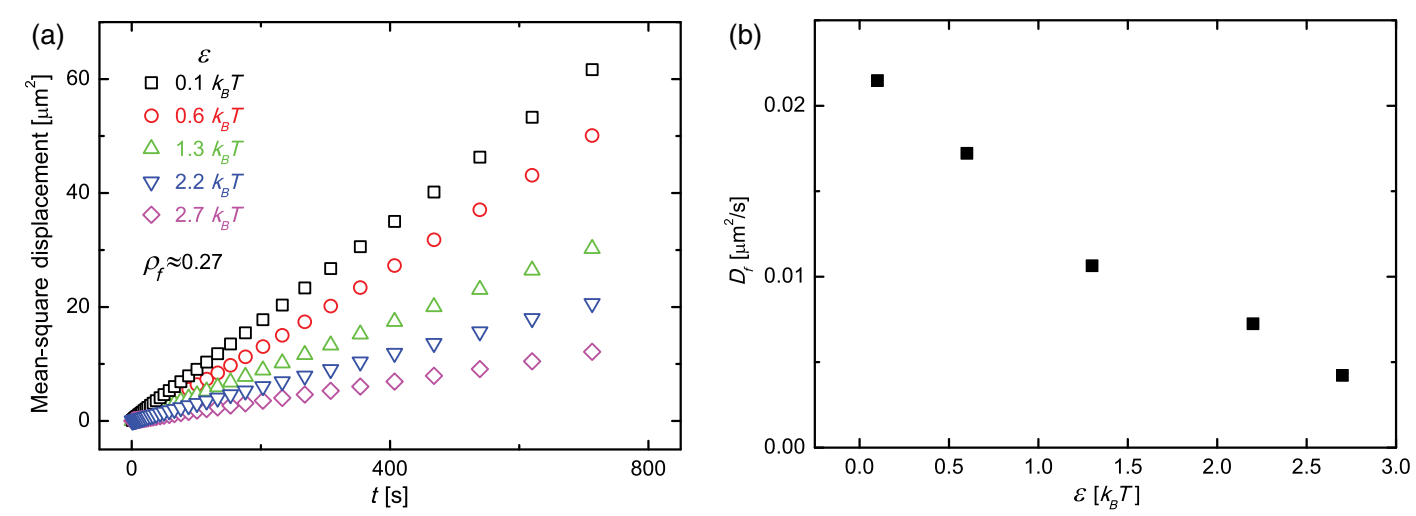

FIG. 8. (a) Mean-square displacement of fluid with $\rho_{f}=0.27$ for short-range systems. The long time-diffusion coefficient $D_{f}$ (b) decays with attraction strength $\varepsilon$, which indicates that the dynamics of fluids slow down as $\varepsilon$ rises. A similar trend is found for fluids at other areal densities and in other long-range systems. Please refer to Appendix C for calculation details.
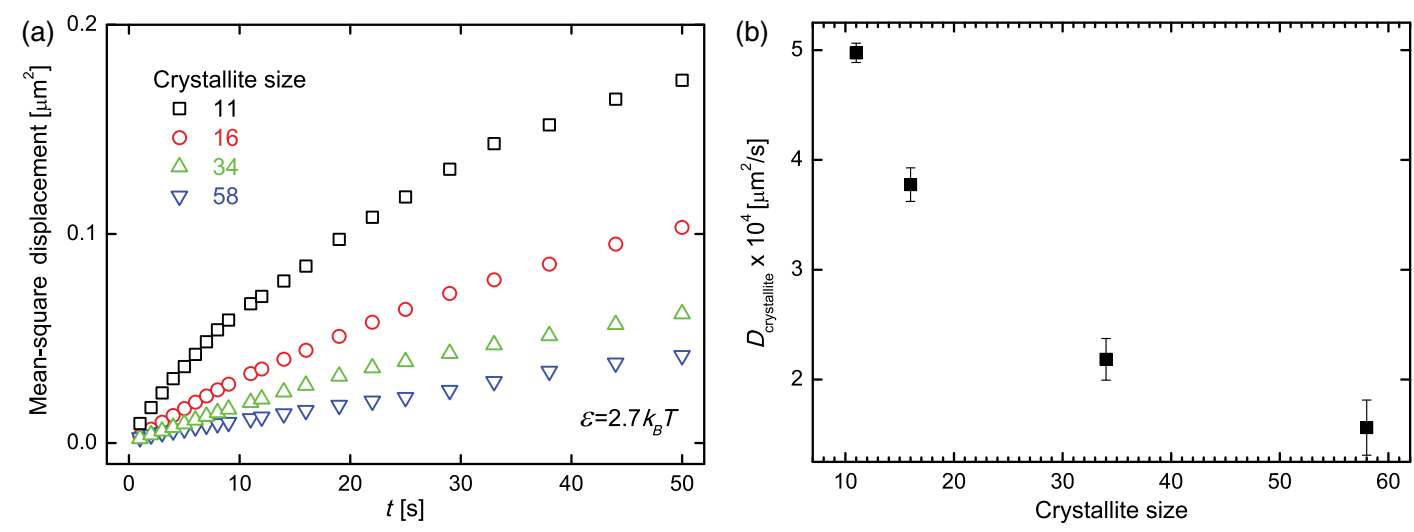

FIG. 9. (a) Mean-square displacement of crystallites as a function of the size at $\varepsilon=2.7 k_{B} T$ for short-range fluid-crystallite coexistence. The long time-diffusion coefficient $D_{\text {crystallite }}$ (b) decays with an increase of the crystallite size until they contact with each other and percolate throughout the system. No splitting or coalescence of crystallite occurs during the measurement (i.e., the size of every crystallite keeps constant during the measurement). Each crystallite contributes to only one diffusion coefficient. The error bar is taken from statistics of different shapes for a given size of the crystallite. Similar behavior is found for crystallites at other $\varepsilon$. Refer to Appendixes C and D for calculation details and identification of crystalline particles and crystallite clusters.

\section{APPENDIX D: IDENTIFICATION OF EACH PHASE FROM THE COEXISTENCE STATE}

\section{Identification of crystalline particles in a fluid or vapor}

Unlike bulk fluid-solid coexistence, where density can be simply extracted in each bulk phase, crystalline particles must be distinguished when they are homogeneously dispersed in a fluid or vapor. By convention, the 2D orientational order parameter $\left|\psi_{6 j}\right| \equiv\left|\left(1 / Z_{j}\right) \sum_{k=1}^{z_{j}} e^{6 i \theta_{j k}}\right|[66]$ is used to identify the crystalline particle, where $\theta_{j k}$ denotes the angle of the bond between reference particle $j$ and its neighbor $k . Z_{j}$ is the number of nearest neighbors identified from the Delaunay triangulation. $\left|\psi_{6 j}\right|=1$ for a particle in a perfect triangular crystalline lattice and 0 for fluids even if $Z_{j}<6$. To exclude transient crystalline particles, we use averaged $\left\langle\left|\psi_{6 j}\right|\right\rangle_{t}>0.8$ over a long period of time $(t=300 \mathrm{~s})$ (Fig. 10). The identification results are almost the same when $t>200 \mathrm{~s}$, where $D_{f}$ is estimated at $\rho_{f}=0.27$ (Fig. 8). As Fig. 10 shows, $\left\langle\left|\psi_{6 j}\right|\right\rangle_{t}$ presents a bimodal distribution, corresponding to fluidlike and crystalline particles, respectively; consequently, we choose 0.8 as the boundary value. The bimodal distribution unambiguously confirms it to be a coexistence state, and, from the locations, two-peak two-phase densities can be reliably estimated [67]. The two bimodals are not totally separated, because interfacial particles on the border of crystallite could transfer between both phase states from time to time. The identification result is consistent with the visual judgment (Fig. 10). Crystallites are defined as follows: If the distance between two nearest crystalline particles is less than $1.5 \sigma$ [3], they are considered to belong to the same crystallite. 

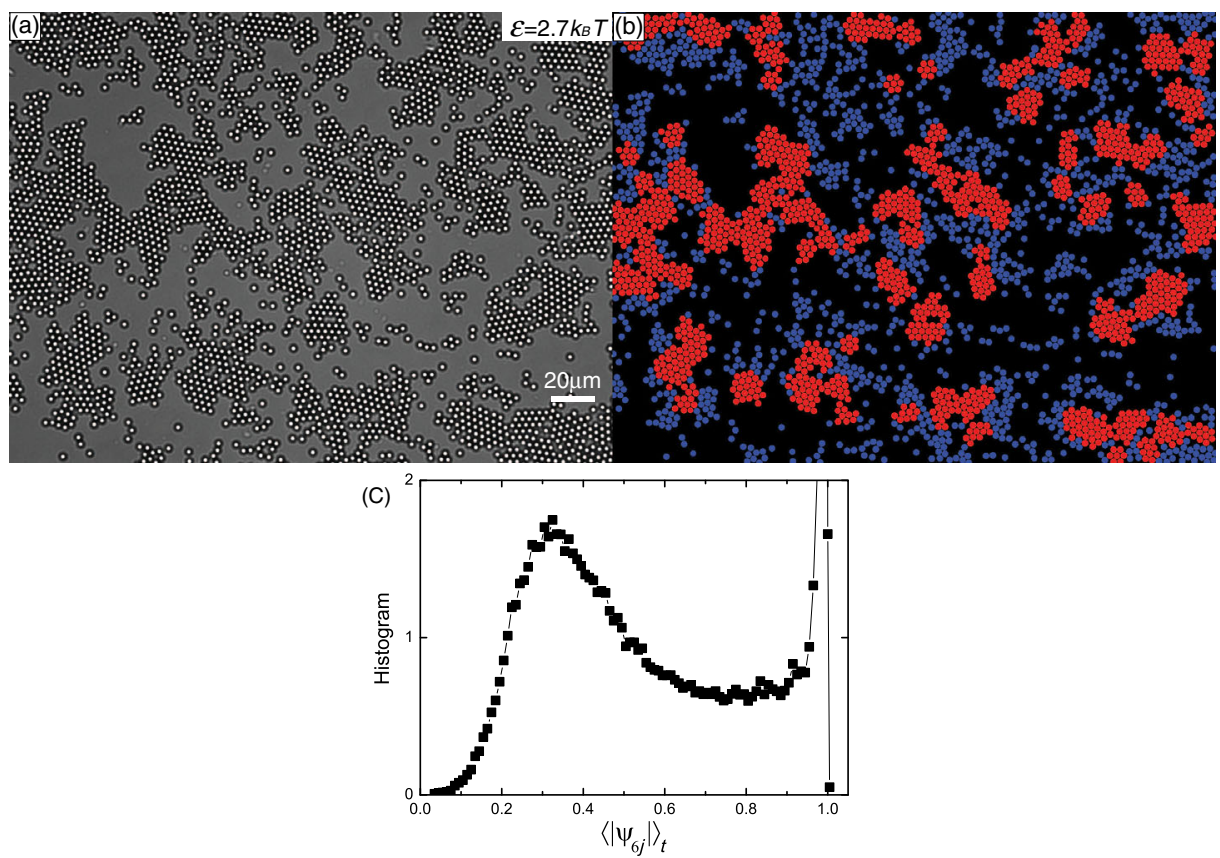

FIG. 10. Identification result for typical crystallite-fluid coexistence at $\varepsilon=2.7 k_{B} T$ in a short-range system. Which phase particle remains can be judged by its local time-averaged orientational order parameter (see Appendix D for details). (a) is the raw image, and (b) is the corresponding colored image for a visualization of identified phases and further density calculation. Blue stands for a fluid particle. Red stands for a crystalline particle. The identification is in good agreement with the visual judgment. (c) The distribution of local time-averaged orientational order parameters exhibits an apparent bimodal distribution, which represents two states (fluid and crystallite).
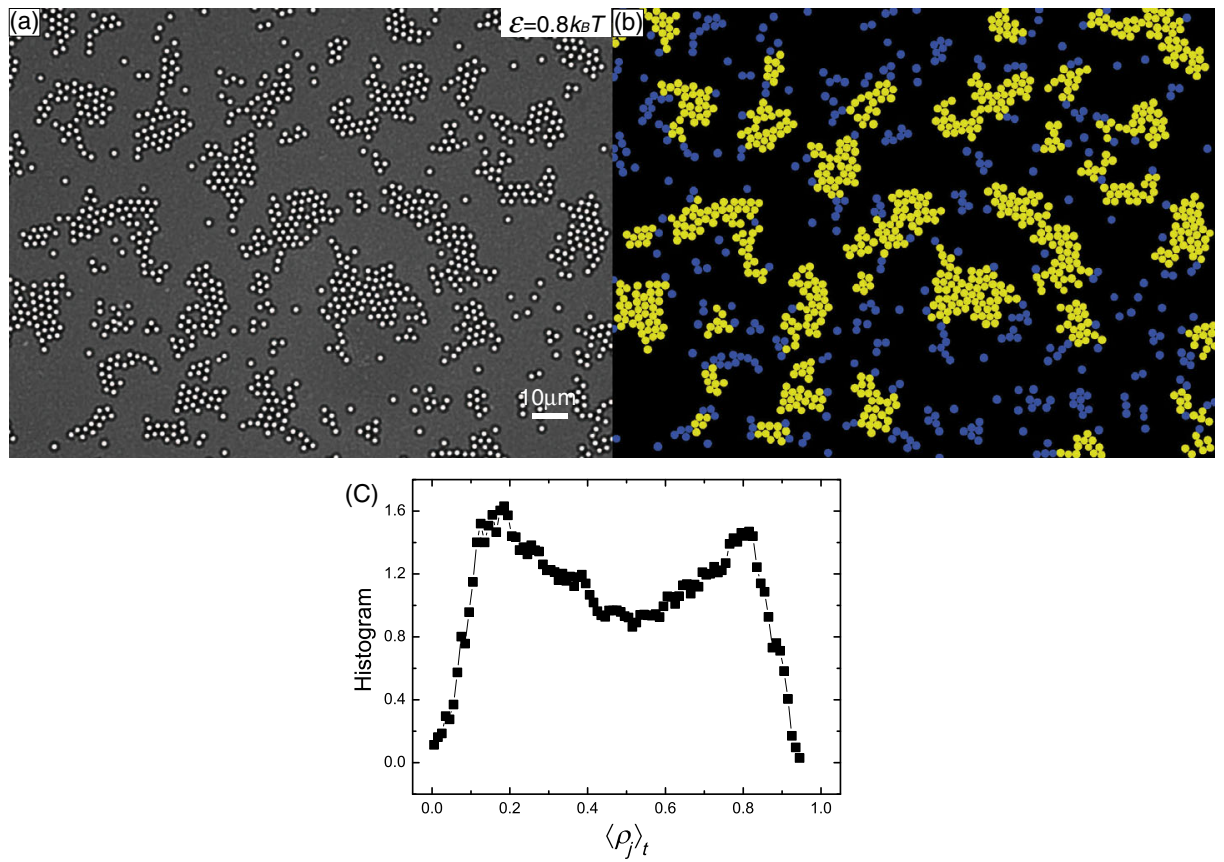

FIG. 11. Identification result for typical vapor-liquid coexistence at $\varepsilon=0.8 k_{B} T$ in a long-range system. Vapor and liquid phases are distinguished by the difference in time-averaged density (see Appendix D for details). (a) is the raw image, and (b) is the corresponding colored image. Yellow denotes liquid particles, while blue denotes vapor particles. (c) The distribution of the time-averaged density for each particle also exhibits a bimodal shape as Fig. 10(c), which confirms the vapor-liquid coexistence. 


\section{Identification of gas and liquid in equilibrium}

Liquid and gas phases are characterized by different densities. Usually, a "liquidlike particle" is defined as having high local density $[3,36]$ during the nucleation process, as is used in analysis of the relaxation process of fluid-fluid into fluid-solid [Fig. 4]. Here, to exclude the transient dense cluster by fluctuation, as is done in the identification of the crystalline particles, we measure the time-averaged $(t=300 \mathrm{~s})$ areal densities of each particle $\left\langle\rho_{i}\right\rangle_{t}$ (Fig. 11) for the equilibrium case. In fact, the identification results are not sensitive when the measured time is larger than 200 s. $\rho \equiv\left[\left(\pi \sigma^{2}\right) / 4 A_{\text {voronoi }}\right]$, where $A_{\text {voronoi }}$ denotes the area of the voronoi cell. The distribution of $\left\langle\rho_{i}\right\rangle_{t}$ (Fig. 11) exhibits a bimodal shape as in Fig. 10. A liquid particle is defined as $\left\langle\rho_{i}\right\rangle_{t} \geq 0.5$, by which value the bimodal distribution is divided. The results of our method are highly consistent with the visual judgment. The particles at the edge of the liquid cluster are carefully counted as liquid particles.

\section{APPENDIX E: QUANTIFICATION OF THE LONG-RANGE POTENTIAL}

Although our long-range potential is different from the Lennard-Jones potential, the attractive part of the potential can be adequately fitted using a power-law function (Fig. 12). The attraction range as well as the power-law form ensures its long-range nature.

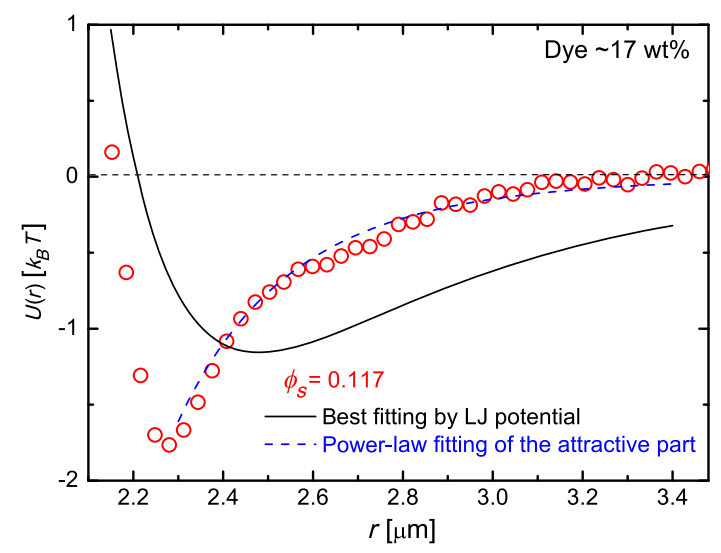

FIG. 12. Solid line: Typical fitting of a long-range potential well at $\varepsilon=1.8 k_{B} T$ by the Lennard-Jones (LJ) formalism $\left(u(r)=4 \varepsilon\left[(\sigma / r)^{12}-(\sigma / r)^{6}\right]\right)$, where fitted $\varepsilon=1.2 k_{B} T$ and $\sigma=2.21 \mu \mathrm{m}$. Dashed line: Power-law function $(u(r)=$ $\left.2 \varepsilon\left[-(\sigma / r)^{9}\right]\right)$ fitting of the attractive part of the long-range potential. The long-distance attractive part of the potential can be well fitted by a power-law function, proving it is indeed long ranged.
[1] F. F. Abraham, The Phases of Two-Dimensional Matter, Their Transitions, and Solid-State Stability: A Perspective via Computer Simulation of Simple Atomic Systems, Phys. Rep. 80, 340 (1981).

[2] K. J. Strandburg, Two-Dimensional Melting, Rev. Mod. Phys. 60, 161 (1988).

[3] P. R. ten Wolde and D. Frenkel, Enhancement of Protein Crystal Nucleation by Critical Density Fluctuations, Science 277, 1975 (1997).

[4] V. J. Anderson and H. N. Lekkerkerker, Insights into Phase Transition Kinetics from Colloid Science, Nature (London) 416, 811 (2002).

[5] D. Frenkel, Colloidal Encounters: A Matter of Attraction, Science 314, 768 (2006).

[6] D. R. Nelson and B. Halperin, Dislocation-Mediated Melting in Two Dimensions, Phys. Rev. B 19, 2457 (1979).

[7] S. C. Kapfer and W. Krauth, Two-Dimensional Melting: From Liquid-Hexatic Coexistence to Continuous Transitions, Phys. Rev. Lett. 114, 035702 (2015).

[8] K. Zahn, R. Lenke, and G. Maret, Two-Stage Melting of Paramagnetic Colloidal Crystals in Two Dimensions, Phys. Rev. Lett. 82, 2721 (1999).

[9] J. Barker, D. Henderson, and F. F. Abraham, Phase Diagram of the Two-Dimensional Lennard-Jones System; Evidence for First-Order Transitions, Physica (Amsterdam) 106A, 226 (1981).

[10] J. Tobochnik and G. Chester, Monte Carlo Study of Melting in Two Dimensions, Phys. Rev. B 25, 6778 (1982).

[11] A. Bakker, C. Bruin, and H. Hilhorst, Orientational Order at the Two-Dimensional Melting Transition, Phys. Rev. Lett. 52, 449 (1984).

[12] C. Udink and D. Frenkel, Orientational Order and SolidLiquid Coexistence in the Two-Dimensional Lennard-Jones System, Phys. Rev. B 35, 6933 (1987).

[13] M. G. Noro and D. Frenkel, Phase Behavior of a Simple Model for Membrane Proteins, J. Chem. Phys. 114, 2477 (2001).

[14] S. Ranganathan, G. Dubey, and K. Pathak, MolecularDynamics Study of Two-Dimensional Lennard-Jones Fluids, Phys. Rev. A 45, 5793 (1992).

[15] S. Chui, Grain-Boundary Theory of Melting in Two Dimensions, Phys. Rev. Lett. 48, 933 (1982).

[16] T. Ramakrishnan, Density-Wave Theory of First-Order Freezing in Two Dimensions, Phys. Rev. Lett. 48, 541 (1982).

[17] B. Joos and M. Duesbery, Dislocation Energies in Rare-Gas Monolayers on Graphite, Phys. Rev. Lett. 55, 1997 (1985).

[18] A. González, Colloidal Crystallization in $2 d$ for ShortRanged Attractions: A Descriptive Overview, Crystals 6, 46 (2016).

[19] M. Santra and B. Bagchi, Line Tension of a Two Dimensional Gas-Liquid Interface, J. Chem. Phys. 131, 084705 (2009).

[20] A. Mitus, A. Patashinski, A. Patrykiejew, and S. Sokolowski, Local Structure, Fluctuations, and Freezing in Two Dimensions, Phys. Rev. B 66, 184202 (2002). 
[21] M. G. Noro and D. Frenkel, Extended Corresponding-States Behavior for Particles with Variable Range Attractions, J. Chem. Phys. 113, 2941 (2000).

[22] H. Lekkerkerker, W.-K. Poon, P. Pusey, A. Stroobants, and P. Warren, Phase Behaviour of Colloid + Polymer Mixtures, Europhys. Lett. 20, 559 (1992).

[23] M. Hagen and D. Frenkel, Determination of Phase Diagrams for the Hard-Core Attractive Yukawa System, J. Chem. Phys. 101, 4093 (1994).

[24] P. G. Vekilov, Nucleation, Cryst. Growth Des. 10, 5007 (2010).

[25] P. G. Vekilov, The Two-Step Mechanism of Nucleation of Crystals in Solution, Nanoscale 2, 2346 (2010).

[26] D. Erdemir, A. Y. Lee, and A. S. Myerson, Nucleation of Crystals from Solution: Classical and Two-Step Models, Acc. Chem. Res. 42, 621 (2009).

[27] W. Ostwald, Studies on the Formation and Change of Solid Matter, Z. Phys. Chem. 22, 289 (1897).

[28] J. F. Lutsko and G. Nicolis, Theoretical Evidence for a Dense Fluid Precursor to Crystallization, Phys. Rev. Lett. 96, 046102 (2006).

[29] T. Schilling, H. J. Schöpe, M. Oettel, G. Opletal, and I. Snook, Precursor-Mediated Crystallization Process in Suspensions of Hard Spheres, Phys. Rev. Lett. 105, 025701 (2010).

[30] A. Lomakin, N. Asherie, and G. B. Benedek, Liquid-Solid Transition in Nuclei of Protein Crystals, Proc. Natl. Acad. Sci. U.S.A. 100, 10254 (2003).

[31] V. Talanquer and D. W. Oxtoby, Crystal Nucleation in the Presence of a Metastable Critical Point, J. Chem. Phys. 109, 223 (1998).

[32] K. G. Soga, J. R. Melrose, and R. C. Ball, Metastable States and the Kinetics of Colloid Phase Separation, J. Chem. Phys. 110, 2280 (1999).

[33] O. Galkin and P. G. Vekilov, Control of Protein Crystal Nucleation around the Metastable Liquid-Liquid Phase Boundary, Proc. Natl. Acad. Sci. U.S.A. 97, 6277 (2000).

[34] O. Galkin, K. Chen, R. L. Nagel, R. E. Hirsch, and P. G. Vekilov, Liquid-Liquid Separation in Solutions of Normal and Sickle Cell Hemoglobin, Proc. Natl. Acad. Sci. U.S.A. 99, 8479 (2002).

[35] O. Gliko, N. Neumaier, W. Pan, I. Haase, M. Fischer, A. Bacher, S. Weinkauf, and P. G. Vekilov, A Metastable Prerequisite for the Growth of Lumazine Synthase Crystals, J. Am. Chem. Soc. 127, 3433 (2005).

[36] J. Savage and A. Dinsmore, Experimental Evidence for Two-Step Nucleation in Colloidal Crystallization, Phys. Rev. Lett. 102, 198302 (2009).

[37] J. Savage, D. Blair, A. Levine, R. Guyer, and A. Dinsmore, Imaging the Sublimation Dynamics of Colloidal Crystallites, Science 314, 795 (2006).

[38] P. N. Pusey and W. Van Megen, Phase Behaviour of Concentrated Suspensions of Nearly Hard Colloidal Spheres, Nature (London) 320, 340 (1986).

[39] J. Zhu, M. Li, R. Rogers, W. Meyer, R. H. Ottewill, W. B. Russel, P. M.Chaikin et al., Crystallization of HardSphere Colloids in Microgravity, Nature (London) 387, 883 (1997).

[40] A. Yethiraj and A. van Blaaderen, A Colloidal Model System with an Interaction Tunable from Hard Sphere to Soft and Dipolar, Nature (London) 421, 513 (2003).
[41] C. Hertlein, L. Helden, A. Gambassi, S. Dietrich, and C. Bechinger, Direct Measurement of Critical Casimir Forces, Nature (London) 451, 172 (2008).

[42] E. Hilou, D. Du, S. Kuei, and S. L. Biswal, Interfacial Energetics of Two-Dimensional Colloidal Clusters Generated with a Tunable Anharmonic Interaction Potential, Phys. Rev. Mater. 2, 025602 (2018).

[43] S. Asakura and F. Oosawa, On Interaction between Two Bodies Immersed in a Solution of Macromolecules, J. Chem. Phys. 22, 1255 (1954).

[44] E. H. de Hoog and H. N. W. Lekkerkerker, Measurement of the Interfacial Tension of a Phase-Separated ColloidPolymer Suspension, J. Phys. Chem. B 103, 5274 (1999).

[45] D. Aarts, R. Tuinier, and H. N. W. Lekkerkerker, Phase Behaviour of Mixtures of Colloidal Spheres and ExcludedVolume Polymer Chains, J. Phys. Condens. Matter 14, 7551 (2002).

[46] H. N. W. Lekkerkerker and R. Tuinier, Colloids and the Depletion Interaction (Springer, New York, 2011).

[47] See Supplemental Material at http://link.aps.org/ supplemental/10.1103/PhysRevX.9.031032 for Movies $1-7$.

[48] Z. Wang, F. Wang, Y. Peng, and Y. Han, Direct Observation of Liquid Nucleus Growth in Homogeneous Melting of Colloidal Crystals, Nat. Commun. 6, 6942 (2015).

[49] D. Frenkel (private communication).

[50] J. Xu and G. Stell, An Analytic Treatment of Percolation in Simple Fluids, J. Chem. Phys. 89, 1101 (1988).

[51] P. N. Segrè, V. Prasad, A. B. Schofield, and D. A. Weitz, Glasslike Kinetic Arrest at the Colloidal-Gelation Transition, Phys. Rev. Lett. 86, 6042 (2001).

[52] A. I. Campbell, V. J. Anderson, J. S. van Duijneveldt, and P. Bartlett, Dynamical Arrest in Attractive Colloids: The Effect of Long-Range Repulsion, Phys. Rev. Lett. 94, 208301 (2005).

[53] T. H. Zhang, J. Groenewold, and W. K. Kegel, Observation of a Microcrystalline Gel in Colloids with Competing Interactions, Phys. Chem. Chem. Phys. 11, 10827 (2009).

[54] F. F. Abraham, Evidence for Two-Dimensional LiquidVapor Coexistence: A Monte Carlo Computer Simulation, J. Chem. Phys. 72, 1412 (1980).

[55] H. Watanabe, N. Ito, and C.-K. Hu, Phase Diagram and Universality of the Lennard-Jones Gas-Liquid System, J. Chem. Phys. 136, 204102 (2012).

[56] B. Smit and D. Frenkel, Vapor-Liquid Equilibria of the Two-Dimensional Lennard-Jones Fluid(s), J. Chem. Phys. 94, 5663 (1991).

[57] X. C. Zeng, Gas-Liquid Nucleation in Two-Dimensional Fluids, J. Chem. Phys. 104, 2699 (1996).

[58] J. A. Blink and W. G. Hoover, Fragmentation of Suddenly Heated Liquids, Phys. Rev. A 32, 1027 (1985).

[59] D. G. A. L. Aarts, M. Schmidt, and H. N. W. Lekkerkerker, Direct Visual Observation of Thermal Capillary Waves, Science 304, 847 (2004).

[60] S. M. Ilett, A. Orrock, W. C. K. Poon, and P. N. Pusey, Phase Behavior of a Model Colloid-Polymer Mixture, Phys. Rev. E 51, 1344 (1995).

[61] J. C. Crocker and D. G. Grier, Methods of Digital Video Microscopy for Colloidal Studies, J. Colloid Interface Sci. 179, 298 (1996). 
[62] D. McQuarrie, Statistical Mechanics (University Science, Sausalito, CA, 2000).

[63] S. H. Behrens and D. G. Grier, Pair Interaction of Charged Colloidal Spheres near a Charged Wall, Phys. Rev. E 64, 050401(R) (2001).

[64] J. Baumgartl and C. Bechinger, On the Limits of Digital Video Microscopy, Europhys. Lett. 71, 487 (2005).
[65] M. Polin, D. G. Grier, and Y. Han, Colloidal Electrostatic Interactions near a Conducting Surface, Phys. Rev. E 76, 041406 (2007).

[66] B. Halperin and D. R. Nelson, Theory of Two-Dimensional Melting, Phys. Rev. Lett. 41, 121 (1978).

[67] K. Mon and K. Binder, Finite Size Effects for the Simulation of Phase Coexistence in the Gibbs Ensemble near the Critical Point, J. Chem. Phys. 96, 6989 (1992). 